\title{
PIONEER VENUS ORBITER MEASUREMENTS OF SOLAR EUV FLUX DURING SOLAR CYCLES 21 AND 22*
}

\author{
K. K. MAHAJAN ${ }^{1}$, HARI OM UPADHYAY ${ }^{1}$, N. K. SETHI ${ }^{1}$, W. R. HOEGY ${ }^{2}$, \\ W. D. PESNELL ${ }^{3}$ and L. H. BRACE ${ }^{4}$ \\ ${ }^{1}$ Radio and Atmospheric Sciences Division, National Physical Laboratory, \\ New Delhi - 110012, India \\ ${ }^{2}$ NASA Goddard Space Flight Center, Greenbelt, MD 20771, U.S.A. \\ ${ }^{3}$ Nomad Research Inc., 2804 Nomard Court, Bowie, MD 20716, U.S.A. \\ ${ }^{4}$ Space Physics Laboratory, University of Michigan, Ann Arbor, MI 48109, U.S.A.
}

(Received 14 October 1996; accepted 2 May 1997)

\begin{abstract}
The Pioneer Venus Orbiter (PVO) had on board the electron temperature probe experiment which measured temperature and concentration of electrons in the ionosphere of Venus. When the probe was outside the Venus ionosphere and was in the solar wind, the probe current was entirely due to solar photons striking the probe surface. This probe thus measured integrated solar EUV flux (Ipe) over a 13-year period from January 1979 to December 1991, thereby covering the declining phase of solar cycle 21 and the rising phase of solar cycle 22. In this paper, we examine the behavior of Ipe translated to the solar longitude of Earth (to be called EIpe) during the two solar cycles. We find that total EUV flux changed by about $60 \%$ during solar cycle 21 and by about $100 \%$ in solar cycle 22 . We also compare this flux with other solar activity indicators such as $F_{10.7}, \mathrm{~L} \alpha$, and the solar magnetic field. We find that while the daily values of EIpe are highly correlated with $F_{10.7}$ (correlation coefficient 0.87 ), there is a large scatter in EIpe for any value of this Earth-based index. A comparison of EIpe with SME and UARS SOLSTICE L $\alpha$ measurements taken during the same period shows that EIpe tracks $L \alpha$ quite faithfully with a correlation coefficient of 0.94 . Similar comparison with the solar magnetic field $(B s)$ shows that EIpe correlates better with $B s$ than with $F_{10.7}$. We also compare EIpe with total solar irradiance measured during the same period.
\end{abstract}

\section{Introduction}

It is now well known that full-disk solar extreme ultraviolet (EUV) irradiances between 1 and $121.6 \mathrm{~nm}$ provide the major energy input to the terrestrial atmosphere. These irradiances are also the primary photoionization source for creating the ionosphere. Therefore for detailed aeronomical studies dealing with the thermal structure and ion distribution in the thermosphere, one needs an accurate knowledge of these irradiances. It is also known that there are both short-term and long-term variations in the EUV flux, and therefore, EUV flux variation in time is one of the fundamental variables in thermospheric and ionospheric physics. Unfortunately, there have been only a few measurements of solar EUV on a daily basis. The various satellite measurements of solar EUV and UV from 1960 onwards have been excellently summarized by Schmidtke (1992). Figure 1 shows these measurements as a function of solar activity. The first measurements were made in 1962 on the

\footnotetext{
* Paper presented at the SOLERS22 International Workshop, held at the National Solar Observatory, Sacramento Peak, Sunspot, New Mexico, U.S.A., June 17-21, 1996.
} 


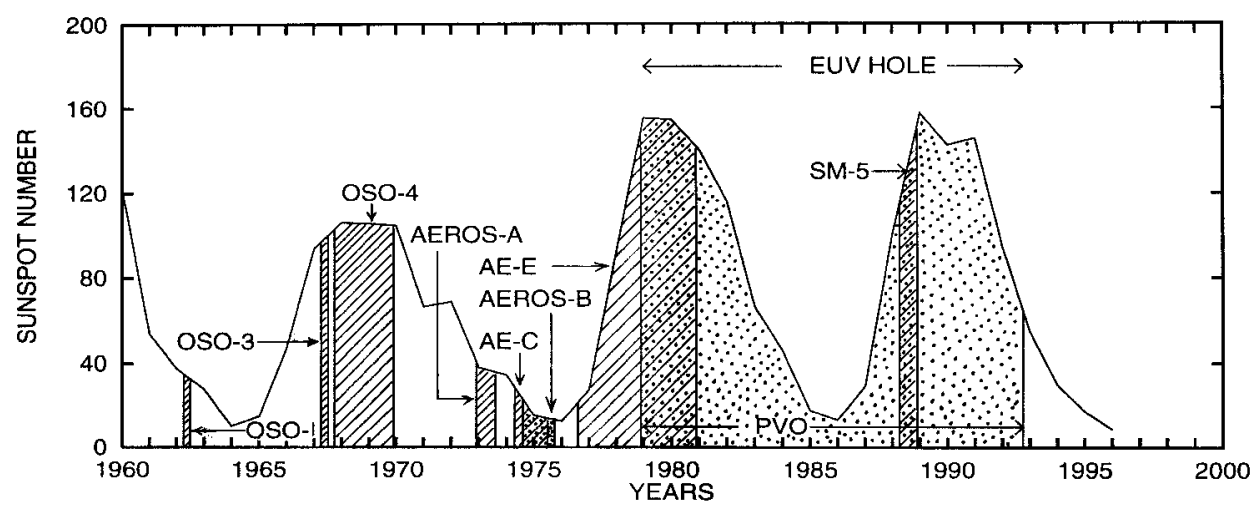

Figure 1. Schematic representation of satellite measurements of solar EUV irradiances from 1960 onwards at various levels of solar activity. PVO measurements of total EUV flux happen to be during the 'EUV hole' period.

OSO-1 satellite (March-May 1962) with an EUV spectrometer in the range 2 $-40 \mathrm{~nm}$ (Lindsay, 1964). The first EUV satellite spectrometer covering the 27$131 \mathrm{~nm}$ wavelength range was operated by Hinteregger and Hall (1969) on OSO-3 (March-June 1967). These measurements provided two important results on the strong variability in the total solar EUV flux: (1) that the solar rotation accounted for $40 \%$ of the variability when the $F_{10.7}$ index increased from 111 to 201 , and (2) the solar cycle variability was about $50 \%$ when the $F_{10.7}$ index increased from 75 to 150 (Schmidtke, 1992). The grating monochrometer experiment aboard OSO-4 (October 1967-December 1969) at $30.4 \mathrm{~nm}$ recorded solar flux variations (Timothy and Timothy, 1970) which showed good agreement with the earlier results. A total flux change of the helium emission $(30.4 \mathrm{~nm})$ for solar cycle 20 was estimated to be about 100\%. The OSO-3 and OSO-4 measurements were followed by AEROS-A (December 1972-August 1973): 16-103 nm, (Schmidtke et al., 1977), AE-C (April 1974-June 1975): 14-185 nm (Hinteregger, 1977), AEROSB (July 1974-September 1975): 16-103 nm (Schmidtke, 1984), AE-E (July 1976 -December 1980): 14-185 nm (Hinteregger, Fukui, and Gilson, 1981) and SM-5 (March-December 1988): 20-700 nm (Schmidtke, Seidl, and Wita, 1985). As can be noted from Figure 1, the longest set of measurements, throughout most of the spectrum, were of the AE-E satellite.

The period 1980 onwards has been named as the 'EUV hole' by Donnelly (1987), because the next full spectrum measurements will not occur until the late 1990s (Tobiska, 1996). Fortunately, during the major portion of the 'EUV hole' period, the Pioneer Venus Orbiter (PVO) carried a Langmuir probe to measure the temperature and concentration of electrons in the ionosphere of Venus. However, when the probe was outside the Venus ionosphere and was in the solar wind, it measured photoelectron current and thus acted as a photodiode. The probe measured integrated solar EUV flux over the 13-year period from January 1979 to December 
1991, thus covering the declining phase of solar cycle 21 and the rising phase of solar cycle 22 . The probe provided fairly accurate values of the EUV flux for a good portion of the 'EUV hole' period, and thus these measurements are of major interest to aeronomers as well as to solar physicists.

The broad features of the PVO EUV flux were first studied by Brace, Hoegy, and Theis (1988) for the declining phase of solar cycle 21. In this paper we extend the analysis to the rising phase of solar cycle 22 and study some fine features of the EUV flux translated to the solar longitude of Earth. These EUV flux measurements are then compared with $L \alpha$ measurements made during the same period on the SME (Rottman, 1988) and UARS satellites (Rottman, Woods, and Sparn, 1993). We also examine the correlation of our PVO measured flux with other Earth-based indices such as $F_{10.7}$ and the solar magnetic field. Finally, we attempt to compare the periodic variations seen in the EUV flux (Hoegy and Wolff, 1989) with those found in the total solar irradiance by Pap and Fröhlich (1992) and Fröhlich (1992) measured by the sensors of the Earth Radiation Budget Experiment (ERBE) of the NIMBUS-7 satellite (Hickey et al., 1988; Hoyt et al., 1992).

\section{The Langmuir Probe Experiment on PVO and the EUV Flux Measurements}

The Langmuir probe experiment aboard the Pioneer Venus Orbiter has been described by Krehbiel et al. (1980). The probe basically consisted of a small boom-mounted metallic cylinder which measured electron and ion currents when immersed in the surrounding ionospheric plasma. There were, however, two independent cylindrical collectors on the PVO; one collector was mounted axially while the other was mounted radially. The two probes were used independently to obtain electron temperature and density of the ionosphere by measuring the current-voltage $(\mathrm{I}-\mathrm{V})$ characteristics.

\subsection{LANGMUIR PROBE AS A PHOTODIODE}

The I-V characteristics measured by the Langmuir probe on the PVO experiment provided a large amount of data on electron density, $N_{e}$, and electron temperature, $T_{e}$, in the ionosphere of Venus. Results of these ionospheric measurements have been reported in several publications. (See reviews by Mahajan and Kar, 1988; Brace and Kliore, 1991; and Kar, 1996, and references therein.) As stated earlier, when the spacecraft was outside the Venus ionosphere, this probe acted as a photodiode. The solar EUV radiations, roughly between 10 and $150 \mathrm{~nm}$, on striking the probe surface, ejected photoelectrons, which produced probe current at the level of 5-20 nA. This current, Ipe, is proportional to the total solar EUV flux for wavelengths which create the ionosphere and heat the thermosphere. A daily value of Ipe was obtained for each pass by selecting the maximum level of Ipe from the measurements taken about one hour before periapsis. Elphic et al. (1984) 
were the first to demonstrate that the photoelectron current, Ipe, measured by the probe could be used as an index of solar EUV flux. They observed solar-rotation related cyclic changes in Ipe coincident with similar cyclic changes in the Venus ionosphere.

The amplitude of the photoelectron current (Ipe), when the collector axis was perpendicular to the Sun, is given by the integral (Brace, Hoegy, and Theis, 1988)

$$
\text { Ipe }=A_{c} e \int_{0}^{\lambda_{t}} \Phi_{\lambda} Y_{\lambda} \mathrm{d} \lambda,
$$

where $A_{c}$ is the area of the collector viewed from the Sun $\left(2.14 \mathrm{~cm}^{2}\right) ; \phi_{\lambda}$, solar emission spectrum; $Y_{\lambda}$, photoelectron yield as a function of wavelength; $\lambda_{t}$, threshold wavelength for emission $(\approx 220 \mathrm{~nm}) ; e$, electron charge.

Brace, Hoegy, and Theis (1988) obtained the following relationship between the total EUV flux and Ipe and gave the name Venus EUV ( $\left.V_{\mathrm{EUV}}\right)$ to this index:

$$
V_{\mathrm{EUV}}=1.53 \times 10^{11} \text { Ipe photons } \mathrm{cm}^{-2} \mathrm{~s}^{-1},
$$

where Ipe is in units of $10^{-9} \mathrm{~A}$.

The dependence of Ipe on wavelength, as calculated from Equation (1) has been studied by Brace, Hoegy, and Theis (1988). The amplitude of the photoelectron current is the product of the photoelectron yield and the solar flux summed over EUV wavelengths between $10 \mathrm{~nm}$ and $150 \mathrm{~nm}$. The relative percentage contribution of the various wavelength regimes to Ipe has been documented by Brace, Hoegy, and Theis (1988), Wolff and Hoegy (1989), Mahajan et al. (1990), and Hoegy et al. (1993). About 55\% of the Ipe is from $\mathrm{L} \alpha$ and $30 \%$ from the $30-110 \mathrm{~nm}$ continuum, at both solar minimum and maximum (Mahajan et al., 1990; Hoegy et al., 1993). The remainder is from strong ionizing lines such as He II, He I, C III, etc. This percentage, too, does not change significantly with solar activity. Hoegy et al. (1993) have estimated that about a 10\% contribution to Ipe is from coronal regions with wavelengths less than $50 \mathrm{~nm}$, the remainder (90\%) is from chromospheric regions with wavelengths more than $50 \mathrm{~nm}$ during solar minimum. During solar maximum these percentages are $14 \%$ and $86 \%$.

The eccentricity of Venus's orbit is 0.0068 , and so the total perihelion to aphelion change is $3 \%$. The orbital variation in Ipe is thus $\pm 1.5 \%$. Since this variation was rather small, no adjustment to the mean Sun-Venus distance was made on this account. Transformation to Earth was done for a constant Sun-Earth distance of 1 AU (i.e., Ipe multiplied by 0.518 ).

\section{The Early Studies of Ipe}

As stated earlier, there were two independent cylindrical collectors for the Langmuir probe experiment. The EUV flux obtained by Elphic et al. (1984) was based on 
measurements by the axial probe during Phase I, the early part (1979-1980) of the mission. This probe had a molybdenum surface. When the fuel reserves ran low, the periapsis was allowed to rise during Phase II (1981-1991) and the instrument was dedicated to the radial probe, which had a rhenium surface. Brace, Hoegy, and Theis (1988), therefore, used radial probe data for the EUV flux. Also the radial probe data turned out to be more useful because of its appropriate mounting location, the greater sensitivity of its electrometer and the more continuous availability of data from this collector over the lifetime of the mission.

The daily average values of photocurrent obtained between 1979 and 1987 were first analysed and published by Brace, Hoegy, and Theis (1988), who found that Ipe exhibited variations related to the solar cycle and solar rotation, as well as to a major 7-month periodicity. They compared Ipe measurements for the year 1981 to 1987 with $F_{10.7}$ and found a good agreement in terms of solar cycle and solar rotation effects, although amplitudes were much larger in $F_{10.7}$. At times when both Venus and Earth were viewing the same solar disc (for example, for the interval near inferior conjunction), the solar rotation components were found to be in phase. These signatures, as expected, were less similar near superior conjunction when Earth and Venus viewed opposite sides of the Sun. A comparison by Brace, Hoegy, and Theis (1988) of Ipe with $\mathrm{L} \alpha$ flux measured by the SME satellite showed a better relationship between these two parameters than with $F_{10.7}$. These Ipe observations were used by Mahajan et al. (1990) to examine the response of the Venus exospheric temperature to changes in solar activity, primarily those related to solar rotation. It was found that Ipe quite faithfully tracked variations in the dayside exospheric temperature. The exospheric temperature was also found to be better correlated with Ipe than with $F_{10.7}$.

Although the early studies used Ipe as an EUV index at Venus, Hoegy and Mahajan (1992) translated the Ipe measurements to the solar longitude of Earth and converted these into EUV flux to provide an index for aeronomical studies at Earth. They called this index $E_{\mathrm{EUV}}$. They studied the behaviour of ionospheric parameters foE, foF1, foF 2 at midlatitude station and compared their relationship with $E_{\mathrm{EUV}}$ and $F_{10.7}$. They found foF1 and foF 2 to be better correlated with $E_{\mathrm{EUV}}$ than with $F_{10.7}$. However, foE was found to be better correlated with $F_{10.7}$, because $F_{10.7}$ is also a proxy for soft X-rays which are an important ionizing source in the E-region. Hoegy and Mahajan also provided a table of the daily values of EUV flux for the period 12 February 1979 through most of 1991.

\section{Features of Total EUV flux (EIpe) during Solar Cycles 21 and 22}

The daily values of Ipe have been translated to solar longitude of Earth (to be called EIpe), by using the following modified transform of Hedin et al. (1983):

$$
\text { EIpe }=0.518\left[\left(29-\Delta t_{d}\right) \operatorname{Ipe}\left(t-\Delta t_{d}\right)+\Delta t_{d} \operatorname{Ipe}\left(t+29-\Delta t_{d}\right)\right] / 29,
$$


where

$$
\Delta t_{d}=\bmod [29(16.08+0.61537 N) / 360,29] .
$$

We have replaced 27, the solar rotation period as seen from Earth in Hedin's transform, with 29, which is the approximate period of rotation of Sun as seen from Venus. The formula $(16.08+0.616537 N)$ is the phase difference in longitude between the Earth and Venus, where $N$ is the orbit number (one orbit per Earth day). The number 0.616537 comes from the phase of superior conjunction which is about 583.9 days. Multiplying this expression by 29/360 converts it to phase of the solar rotation in days where 29 is one full cycle as seen from Venus. Thus $\Delta t_{d}$ is the phase difference between Earth and Venus, so days $t-\Delta t_{d}$ and $t+\left(29-\Delta t_{d}\right)$ are the closest days when the solar flux measured on Venus is at the longitude of the Sun facing the Earth on day $t$. This is not perfect because the given longitude of the Sun does not preserve its flux during the 29-day rotation, so we average these closest two observations with a weight corresponding to the time difference of the observations, i.e., $\left(29-\Delta t_{d}\right) / 29$ and $\Delta t_{d} / 29$, respectively. Basically it is a symmetric transformation with an assumption of a 27-day rotation period for solar EUV as seen from Earth.

The daily values of EIpe so obtained are shown in Figure 2 for the period January 1979 to December 1991. This 13-year period covers the peak and later stages of solar cycle 21, the intervening minimum and the peak of solar cycle 22 . A major feature is the large variance in EIpe on a day-to-day basis during solar maxima (1980 to 1982 and 1990 to 1992). This variance is rather small during the solar minimum (1984 to 1987). In addition, there are the well-known solar rotation and solar cycle effects. Using the values of EIpe during the solar minimum years 1985 and 1986, one notes a solar cycle change from 1.6 in cycle 21 to nearly 2.0 in cycle 22. As we shall see later, this is consistent with the SME and UARSSOLSTICE L $\alpha$ measurements. Another feature is the double maxima in EIpe in solar cycle 22, with the second maximum higher than the first maximum. The double maxima can be seen in $F_{10.7}$ also, but here the second maximum is slightly smaller than the first maximum. In addition to these well-known solar rotation and solar cycle changes, a 7-month periodicity can also be noted (Brace, Hoegy, and Theis, 1988; Hoegy and Wolff, 1989). The solar-cycle effect and the 7-month periodicity can be better appreciated if one examines 81-day averaged values of EIpe, as demonstrated in Figure 3.

\subsection{COMPARISON WITH L $\alpha$, SOLAR MAGNETIC FIELD, $F_{10.7}$, AND TOTAL SOLAR IRRADIANCE}

During the period when PVO made the total solar EUV flux measurements, SME and UARS-SOLISTICE instruments provided the L $\alpha$ intensity and NIMBUS-7 the total solar irradiance. In addition, two more Earth-based solar activity indices were available during that period; these are the Covington index $F_{10.7}$, and the 


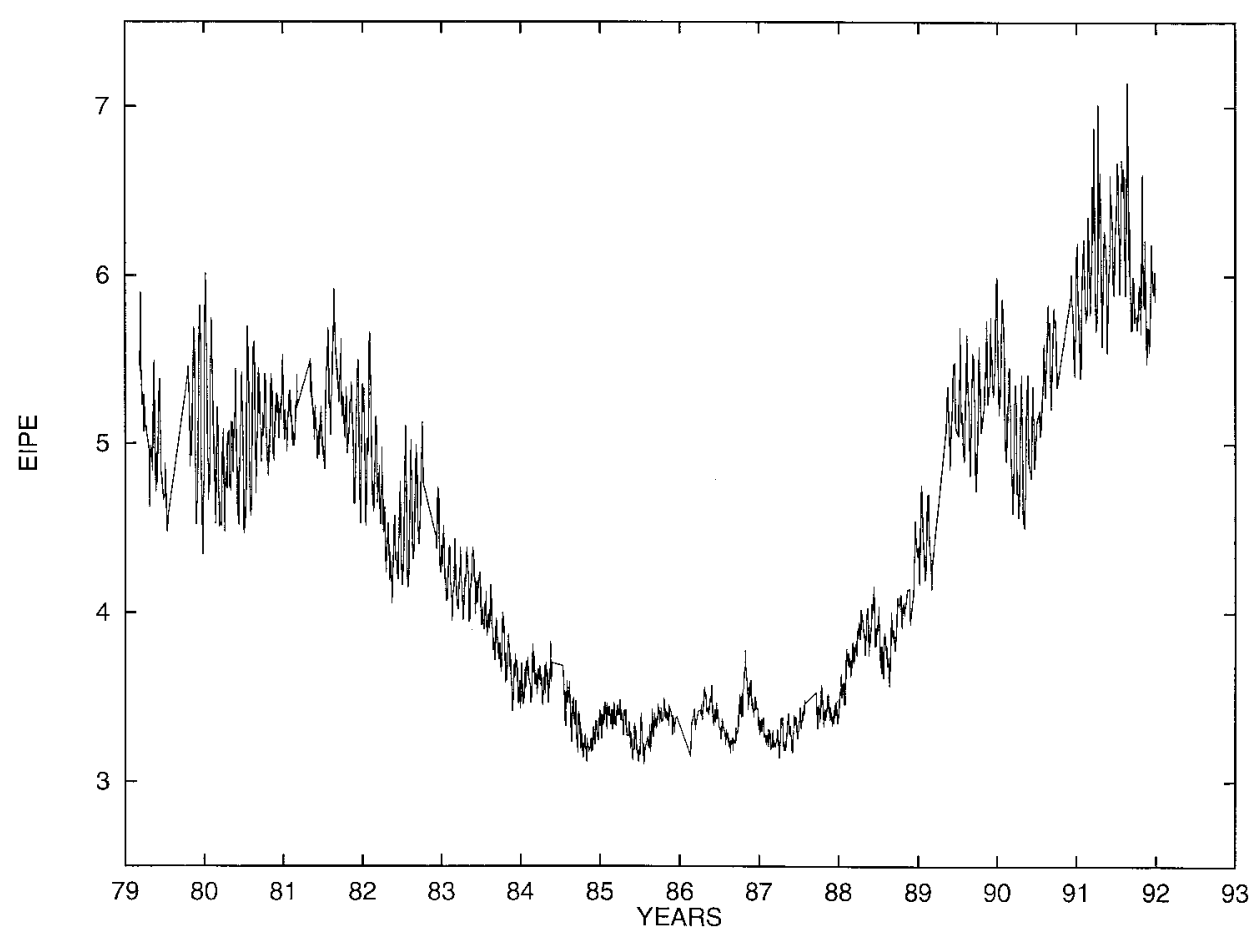

Figure 2. A plot of daily values of EIpe for the period January 1979 to December 1991. The period covered the declining part of solar cycle 21 and rising part of solar cycle 22. The units for EIpe are $10^{-9} \mathrm{~A}$.

solar magnetic field (see, e.g., Harvey, 1992). $F_{10.7}$ has long been used as an EUV index for aeronomical studies. However, solar magnetic field has been recently exploited as an index for solar physics and aeronomical studies. These fields have been measured since 1975 and are determined from the daily, spatially-resolved full-disk magnetograms, and data are now available via ftp of a file from National Solar Observatory / Kitt Peak.

It is now known that the solar atmosphere is highly structured by magnetic fields, and the variability of these fields is the primary modulator of the solar irradiance on time scales of hours to years (Harvey, 1992). In a detailed study of the solar magnetic fields during solar cycles 21 and 22, Harvey (1992) has concluded that the quiet component of the field increases by a factor of 2 or less during a solar cycle, while the active component increases by more than 20 times. She also found that the total solar magnetic flux and the active-region magnetic fields averaged over Carrington rotations correlated well with total solar irradiance, L $\alpha$ intensity, 1-8 X-ray flux, He I $10830 \AA$ equivalent width and $F_{10.7}$, with correlation coefficients of $0.939,0.938,0.896,0.948$, and 0.981 , respectively. We have, therefore, compared our EUV flux with $\mathrm{L} \alpha$, solar magnetic field, $F_{10.7}$ and total solar irradiance in the left-hand panel of Figure 4, which shows a plot of daily values of these parameters. 


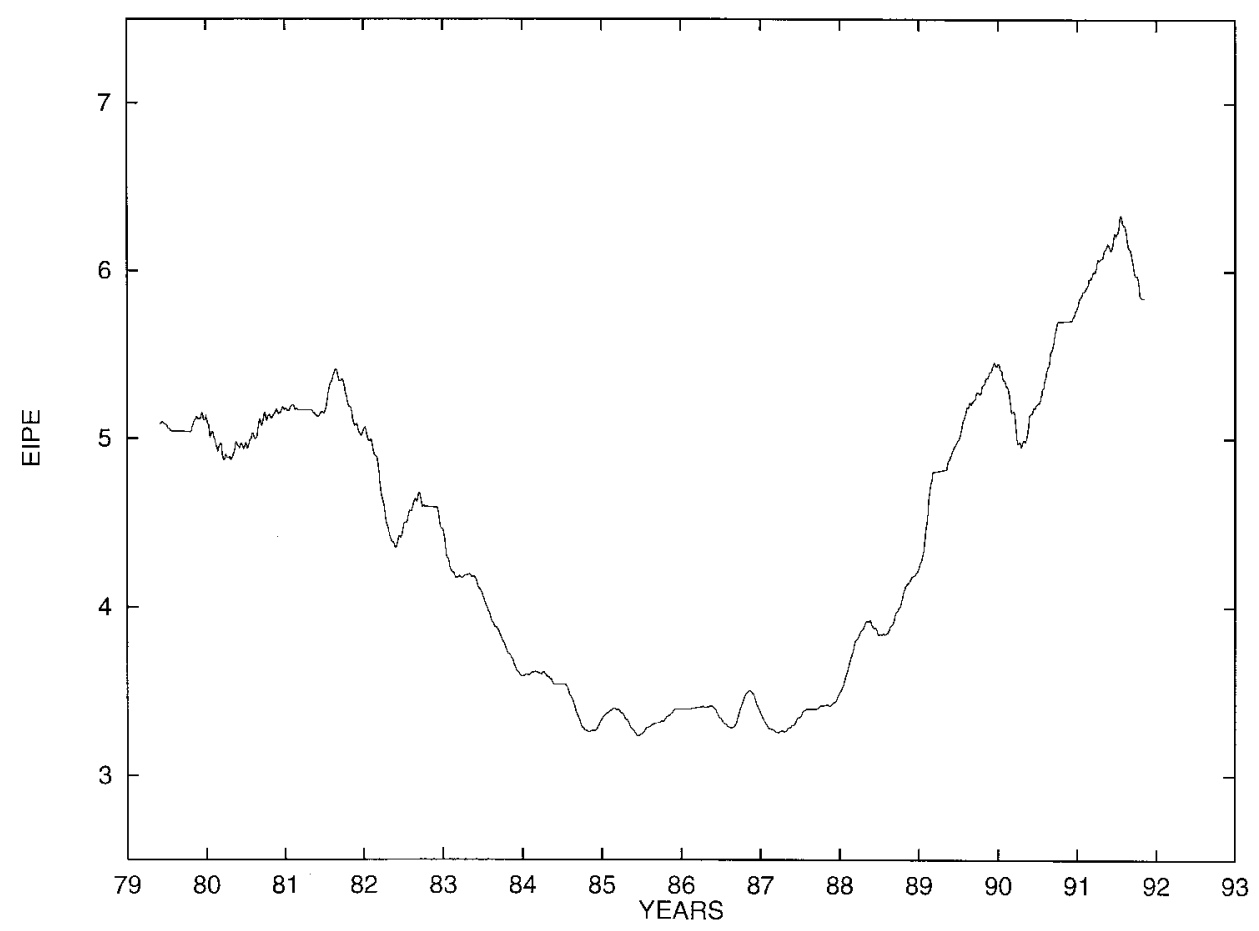

Figure 3. A plot of 81-day averaged EIpe. Long-term periodicities can be noted.

The L $\alpha$ values are taken from the SME and UARS-SOLSTICE (Rottman, 1993) instruments. To bring agreement between SME and UARS measurements, the SME values have been scaled up by $25 \%$ (Woods and Rottman, 1997). The solar magnetic field data is taken from the NSO measurements. It can be noted that like EIpe, all the parameters show large variance on a day-to-day basis during solar maxima. The variance is much smaller during solar minimum. Further, solar cycle effects seen in EIpe agree with those seen in all the other parameters. However, the amplitudes are the largest for $F_{10.7}$ and for solar magnetic field but smaller for $\mathrm{L} \alpha$, EIpe and total solar irradiance. It is to noted also that between 1985 and 1987 when EIpe, solar magnetic field, $F_{10.7}$, and total solar irradiance had become nearly constant and reached their minimum values, the $L \alpha$ was still showing a decreasing trend. This is a very surprising result, and a possible cause could be a change in the sensitivity of the SME instrument. We are tempted to believe this to be the case because all the other four measurements (viz., EIpe, solar magnetic field, $F_{10.7}$, and total solar irradiance) showed similar trends.

Multi-variate spectral analysis of the total solar irradiance by Pap and Fröhlich (1992) and by Fröhlich (1992) have shown variations over time scales from minutes to years and decades with most important variance in the range from days to several months. They have found these variations to be related to photospheric features of 


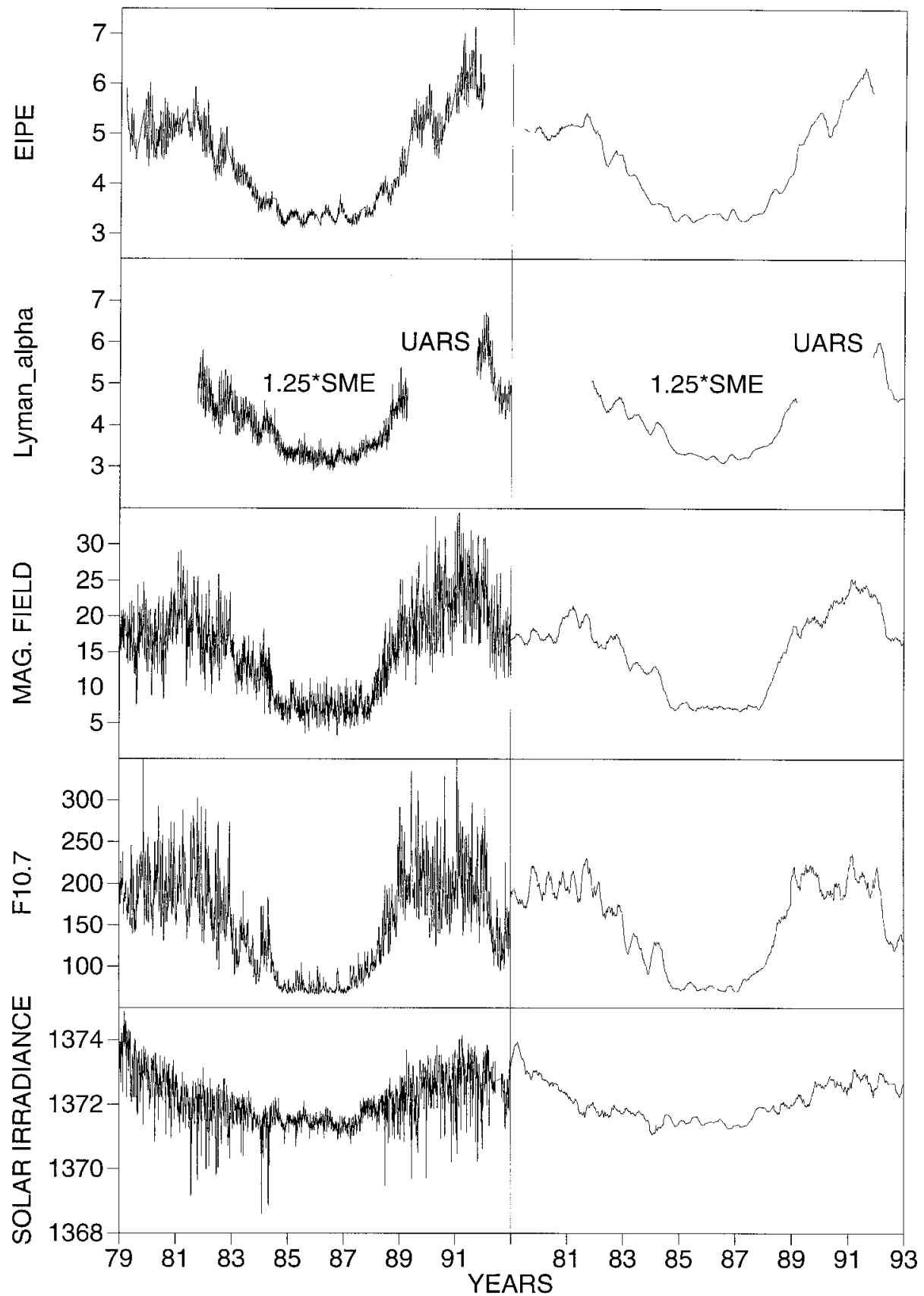

Figure 4. A comparison of time series of EIpe, L $\alpha$, solar magnetic field, $F_{10.7}$, and total solar irradiance. Units for EIpe: $10^{-9} \mathrm{~A}$; for $\mathrm{L} \alpha: 10^{11}$ photon $\mathrm{cm}^{-2} \mathrm{~s}^{-1}$; for solar magnetic field: gauss; for $F_{10.7}: 10^{-22} \mathrm{~W} \mathrm{~m}^{-2} \mathrm{~Hz}^{-1}$; for total solar irradiance: $\mathrm{W} \mathrm{m}^{-2}$. Daily values (left hand panel), 81-day averaged values (right-hand panel). 


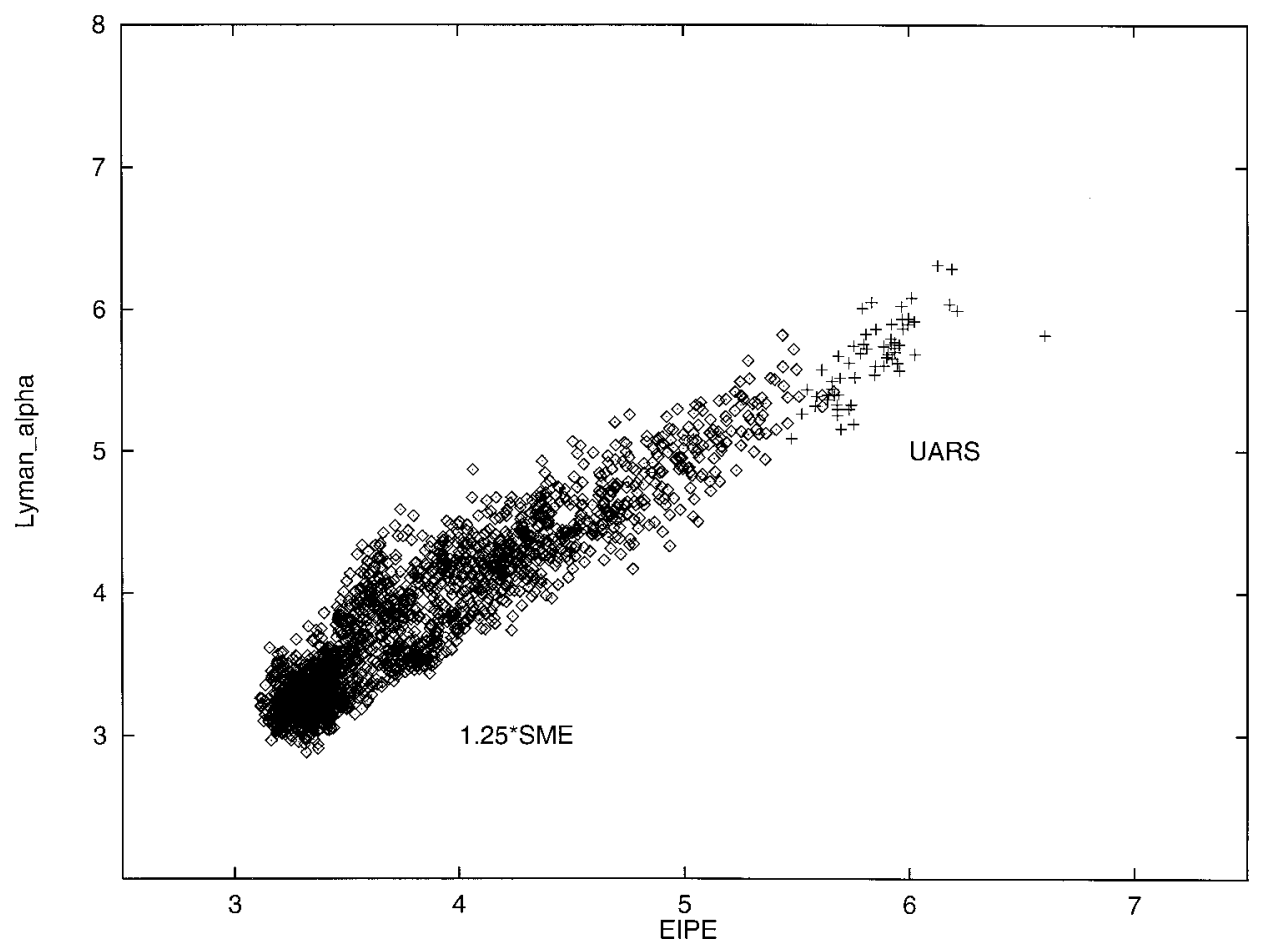

Figure 5. A scatter plot of daily values of EIpe and L $\alpha$. SME measurements have been scaled up by $25 \%$. Units of EIpe: $10^{-9} \mathrm{~A} ; \mathrm{L} \alpha: 10^{11}$ photons $\mathrm{cm}^{-2} \mathrm{~s}^{-1}$.

solar activity; decreased irradiance when sunspots appear and increased irradiance by faculae and the magnetic network. Hoyt et al. (1992) have concluded that for yearly averages the irradiance varies by $0.15 \%$ of the mean, for monthly means it is $0.25 \%$, for daily means, it is $0.46 \%$ and for decadal means it is estimated to be $0.09 \%$. Spectral analysis of Ipe by Hoegy and Wolff (1989) have shown that the dominant periods are the 7-month period at 216 days and the solar rotation at 28 days. The 7-month periodicity can be seen more clearly in Figure 4 (right-hand panel) when 81-day averaged values are plotted.

Another important feature to be noted in Figure 4 is that from the beginning of 1989 to the end of 1991 , while $F_{10.7}$ had nearly reached its maximum value and had leveled off, both EIpe and solar magnetic field were still increasing. A double maxima, however, is seen in all the four parameters during the maximum of solar cycle 22. Unfortunately, there was a large observational gap in the $\mathrm{L} \alpha$ measurements during the rising part of solar cycle 22, but the second maximum seen in the other four parameters is also present in $L \alpha$. 


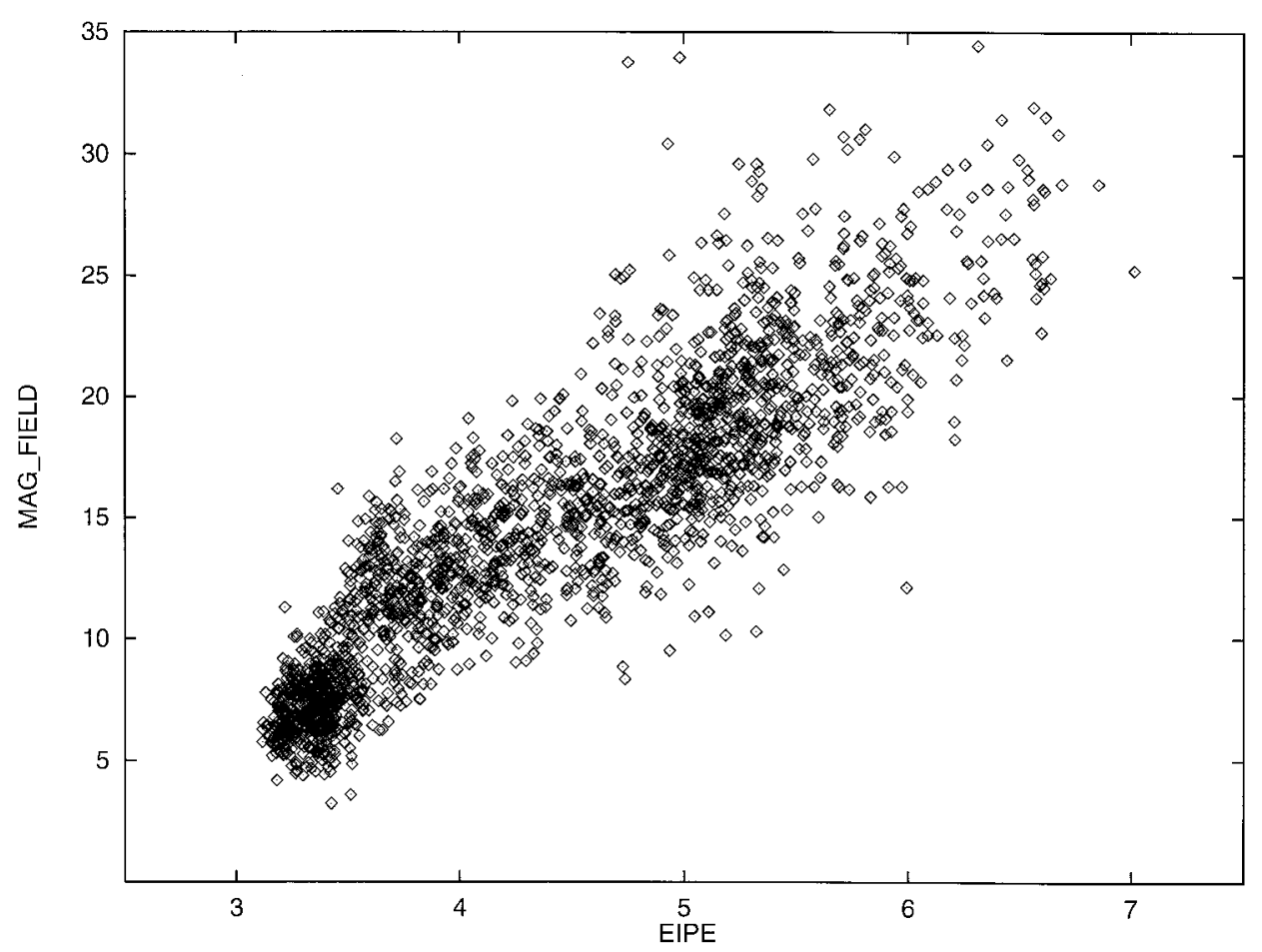

Figure 6. A scatter plot of daily values of EIpe and solar magnetic field. Units of EIpe: $10^{-9} \mathrm{~A}$; solar magnetic field: gauss.

\subsection{Correlation OF EIPE WITH L $\alpha$, SOlAR MAGNETIC FIELD, $F_{10.7}$, AND TOTAL} SOLAR IRRADIANCE

Figures 5-7 show plots of daily values of EIpe against daily values of $L \alpha$, solar magnetic field and $F_{10.7}$, respectively. We find a strong correlation between EIpe and $\mathrm{L} \alpha$, with a correlation coefficient of 0.94 . This is not a surprising result because EIpe is basically a index of chromospheric emissions and 55\% of the contribution of EIpe is from L $\alpha$. The EIpe-solar magnetic field plot shows a larger scatter when compared to the EIpe-L $\alpha$ plot, though the relationship is nearly linear. The correlation coefficient is 0.90 . In the plot between daily values of EIpe and $F_{10.7}$, the scatter is considerably larger compared to the other two plots. However, the correlation coefficient, in spite of the large scatter, is 0.87 . This scatter is mainly due to higher excursions of $F_{10.7}$ during solar rotation, especially during solar maximum, and is mainly due to short-lived boosts from coronal emissions (Donnelly, 1993, private communication). It has already been established that the 27-day variations of $F_{10.7}$ and chromospheric emissions are different (Wolff and Hoegy, 1989; Lean, 1987; Barth et al., 1990). 


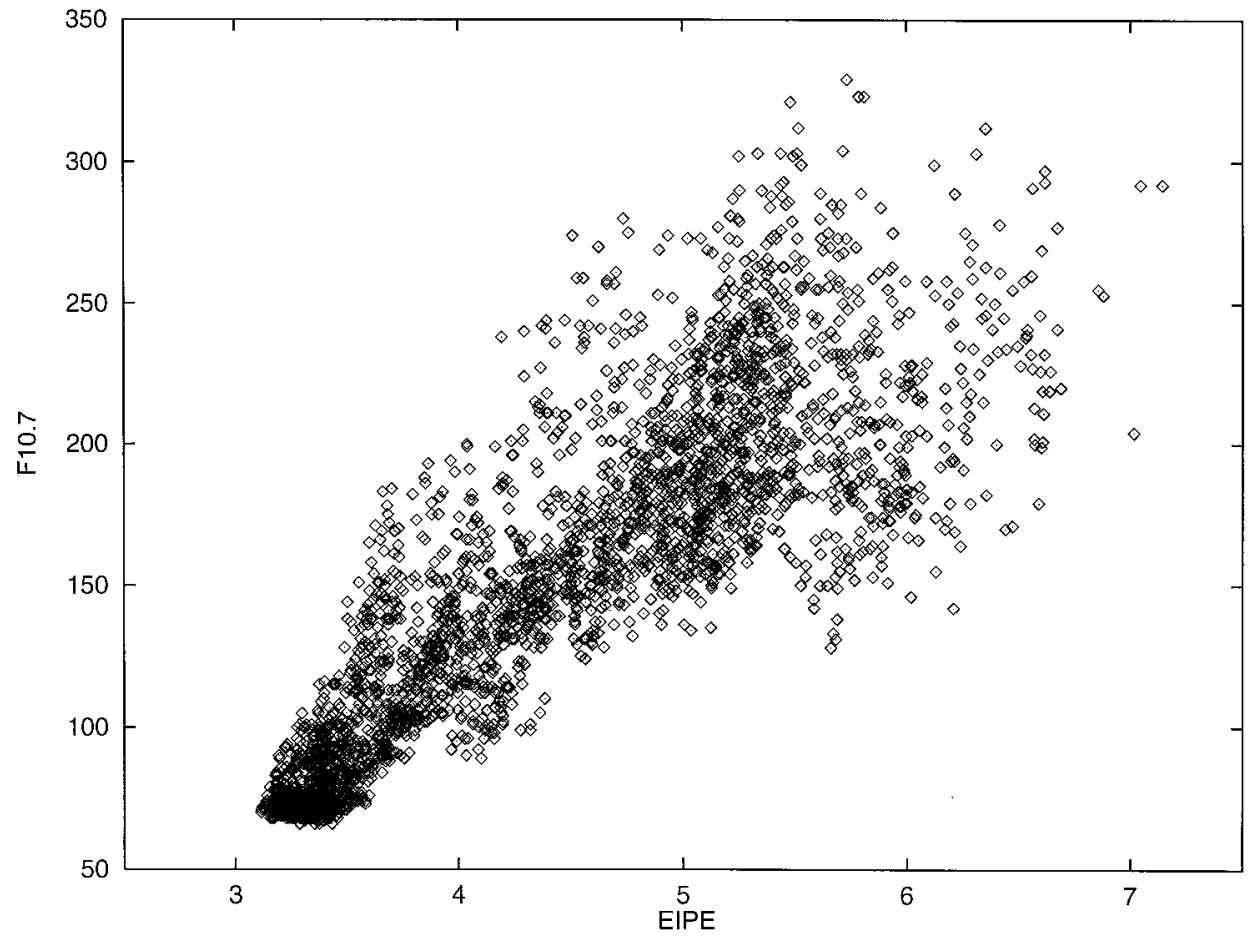

Figure 7. A scatter plot of daily values of EIpe and $F_{10.7}$. Units of EIpe: $10^{-9} \mathrm{~A} ; F_{10.7}$ : $10^{-22} \mathrm{~W} \mathrm{~m}^{-2} \mathrm{~Hz}^{-1}$.

Finally, daily values of EIpe are plotted against total solar irradiance in Figure 8. The correlation coefficient is 0.59 . Higher values of correlation coefficient have been obtained by Harvey (1992) while studying the relationship between total solar irradiance and solar magnetic field, because she used values averaged over one Carrington rotation.

\section{Discussion and Conclusion}

The integrated EUV flux measured by the Langmuir probe on the spacecraft Pioneer Venus Orbiter during the 13-year period January 1979 to December 1991 has shown the well-known solar cycle and solar rotation effects. There is a significant variance in daily flux of all the solar parameters, which makes it difficult to compare daily Earth-based observations with the Venus observations that have been translated in solar longitude; in addition, there are differences in solar flux at different solar longitudes that could, for example, be seen at Venus and not at Earth and vice versa. The largest variance occurs during solar maximum, which is also seen in solar $\mathrm{L} \alpha$, solar magnetic field, $F_{10.7}$, and total solar irradiance. The solar cycle change in total solar EUV flux was about $60 \%$ in cycle 21 and about $100 \%$ in cycle 22 . 


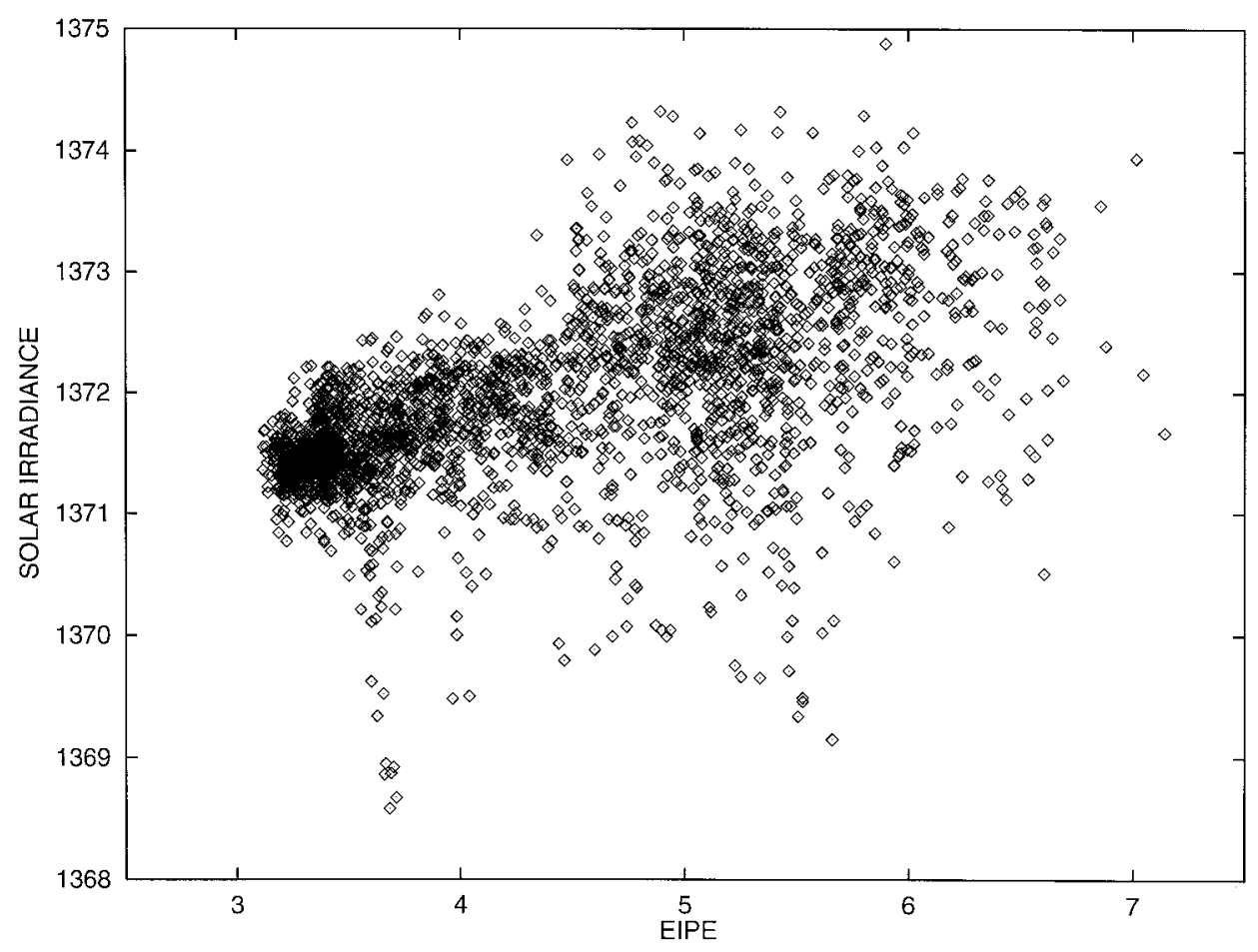

Figure 8. A scatter plot of daily values of EIpe and total solar irradiance. Units of EIpe: $10^{-9} \mathrm{~A}$; total solar irradiance: $\mathrm{W} \mathrm{m}^{-2}$.

\section{Acknowledgements}

We thank Joe Johnston for his diligent production of the Ipe data, T. N. Woods and G. J. Rottman for providing the L $\alpha$ data, K. H. Harvey for the solar magnetic field data, Pat Hrubiak for total solar irradiance data, and Amar Singh for secretarial help. NSO/Kitt Peak magnetic field data used here are produced cooperatively by NSF/NOAO, NASA/GSFC, and NOAA/SEL. H.O.U. held a Senior Research Associateship of the Council of Scientific and Industrial Research, New Delhi, India, during the progress of this work. Finally, we are highly grateful to the referee of this paper, who provided very exhaustive and helpful comments.

\section{References}

Barth, C. A., Tobiska, W. K., Rottman, G. J., and White, O. R.: 1990, Geophys. Res. Letters 17, 571. Brace, L. H. and Kliore, A. J.: 1991, Space Sci. Rev. 55, 81.

Brace, L. H., Hoegy, W. R., and Theis, R. F.: 1988, J. Geophys. Res. 93, 7282.

Donnelly, R. F.: 1987, in P. Foukal (ed.), Solar Radiative Output Variation, Cambridge Research and Instrumentation, Cambridge, Mass., p. 139.

Elphic, R. C., Brace, L. H., Thesis, R. F., and Russell, C. T.: 1984, Geophys. Res. Letters 11, 124. 
Fröhlich, C.: 1992, in R. F. Donelly (ed.), Proceedings of the Workshop on the Solar Electromagnetic Radiation Study for Solar Cycle 22, SEL, NOAA, Boulder, CO, p. 1.

Harvey, K. L.: 1992, in R. F. Donelly (ed.), Proceedings of the Workshop on the Solar Electromagnetic Radiation Study for Solar Cycle 22, SEL, NOAA, Boulder, CO, p. 113.

Hedin, A. E., Niemann, H. B., Kasprzak, W. T., and Seiff, A.: 1983, J. Geophys. Res. 88, 73.

Hickey, J. R., Alton, B. M., Kyle, H. L., and Major, E. R.: 1988, Adv. Space Res. 8 (7), 5.

Hinteregger, H. E.: 1977, Geophys. Res. Letters 4, 231.

Hinteregger, H. E. and Hall, L. A.: 1969, Solar Phys. 6, 175.

Hinteregger, H. E., Fukui, K., and Gilson, B. R.: 1981, Geophys. Res. Letters 8, 1147.

Hoegy, W. R. and Wolff, C. L.: 1989, J. Geophys. Res. 94, 8663.

Hoegy, W. R. and Mahajan, K. K.: 1992, J. Geophys. Res. 97, 10525.

Hoegy, W. R., Pesnell, W. D., Woods, T. N., and Rottman, G. J.: 1993, Geophys. Res. Letters 13, 1335.

Hoyt, D. V., Kyle, H. L., Hickey, J. R., and Maschoff, R. H.: 1992, J. Geophys. Res. 97, 51.

Kar, J.: 1996, Space Sci. Rev. 77, 193.

Krehbiel, J. P., Brace, L. H., Theis, R. F., Cutler, J. R., Pinkus, W. H., and Kaplan, R. B.: 1980, IEEE Trans. Geosci. Remote Sens. GE-18, 49.

Lean, J.: 1987, J. Geophys. Res. 92, 839.

Lindsay, J. C.: 1964, Planetary Space Sci. 12, 379.

Mahajan, K. K. and Kar, J.: 1988, Space Sci. Rev. 47, 303.

Mahajan, K. K., Kasprzak, W. T., Brace, L. H., Niemann, H. B., and Hoegy, W. R.: 1990, J. Geophys. Res. 95, 1091.

Pap, J. M. and Fröhlich, C.: 1992, in R. F. Donelly (ed.), Proceedings of the Workshop on the Solar Electromagnetic Radiation Study for Solar Cycle 22, SEL, NOAA, Boulder, CO, p. 62.

Rottman, G. J.: 1988, Adv. Space Res. 8 (7), 53.

Rottman, G. J., Woods, T. N., and Sparn, T. P.: 1993, J. Geophys. Res. 98, 10667.

Schmidtke, G.: 1984, in K. Rawer (ed.), Encylopedia of Physics XLIX/7, Geophysics III, Part VII, p. 1.

Schmidtke, G.: 1992, in R. F. Donelly (ed.), Proceedings of the Workshop on the Solar Electromagnetic Radiation Study for Solar Cycle 22, SEL, NOAA, Boulder, CO, p. 303.

Schmidtke, G., Seidl, P., and Wita, C.: 1985, Appl. Optics 24, 3206.

Schmidtke, G., Rawer, K., Botzek, H., and Holzer, K.: 1977, J. Geophys. Res. 92, 2423.

Timothy, A. F. and Timothy, J. G.: 1970, J. Geophys. Res. 75, 6950.

Tobiska, W. T.: 1996, Adv. Space Res. 18 (3), 3.

Wolff, C. L. and Hoegy, W. R.: 1989, Solar Phys. 123, 7.

Woods, T. N. and Rottman, G. J.: 1997, J. Geophys. Res. 102, 8769. 